PLANTADANINHA I(2):43-48, 1978

\title{
COMPETIÇÃO ENTRE QUATRO HERBICIDAS INDICADOS PARA O CONTROLE DE PLANTAS DANINHAS EM CANA-DE-AÇÚCAR (Saccharum sp)
}

\author{
* L.S.P. Cruz e L. Leiderman
}

* Pesquisadores Científicos do Instituto Biológico. Seção de Herbicidas. Caixa Postal $70-13.100$ Campinas, SP.

Bolsistas do CNPq

Trabalho apresentado no $24 .^{\circ}$ Congresso Nacio nal de Botânica, realizado em janeiro de 1973 em Pelotas, RS.

Recebido para publicação em 25/maio/78

\section{RESUMO}

Com a finalidade de estudar a ação dos herbicidas oxadiazon, napropamide, methazole e A-3587 na cultura de cana-de-açúcar (Saccharum sp), foram conduzidos em 1971/72 dois ensaios de campo nos municípios paulistas de Capivari e Leme.

Os herbicidas acima citados foram comparados, em trés doses, com 2,4-D anina e atrazine, aplicados em pré-emergência.

Oxadiazon a $2,0 \mathrm{~kg} /$ ha e A-3587 a $3,0 \mathrm{~kg}$ e $4,0 \mathrm{~kg} / \mathrm{ha}$ foram eficientes no controle das principais plantas daninhas que apareceram nos experimentos: capim-marmelada, Brachiaria plantaginea (Link) Hitch; capim-de-colchão, Digitaria sanguinalis (L.) Scop; capim-pé-degalinha, Eleusine indica (L.) Gaertn.; beldroega, Portulacca oleracea L e guanxumas, Sida spp. Napropamide a $3,0 \mathrm{~kg} /$ ha e methazole a $3,5 \mathrm{~kg}$ e $4,0 \mathrm{~kg} /$ ha só não con trolaram as guanxumas.
Nas doses empregadas, nenhum dos herbicidas mostrou-se prejudicial às plantas e à produção de cana-de-açúcar.

\section{SUM MARY}

\section{COMPETITION ON FOUR HERBICIDES FOR CONTROLING WEEDS IN SUGARCANE (Saccharum sp)}

This paper reports on two field trials carried out during 1971/72, in Capivari and Leme, with the herbicides oxadiazon, napropamide, methazole and A-3587, in sugar-cane crops.

In these experiments the four herbicides, in three doses, were compared, in preemergence, with 2,4-D amine and atrazine.

Oxadiazon at $2.0 \mathrm{~kg} / \mathrm{ha}$ and $\mathrm{A}-3587$ at $3.0 \mathrm{~kg}$ and $4,0 \mathrm{~kg} /$ ha gave good control against Brachiaria plantaginea, Digitaria sanguinalis, Eleusine indi- 
ca, Portulacca oleracea and Sida spp, wich were found in the trials. Napropamide at $3.0 \mathrm{~kg} / \mathrm{ha}$ and methazole at $3.5 \mathrm{~kg}$ and $4.0 \mathrm{~kg} / \mathrm{ha}$ gave good control against those weeds but except Sida spp. None of the herbicides caused injuries to the crop or reduced yield.

\section{INT ROD UÇÃO}

O uso de herbicidas no controle de plantas daninhas em cana-deaçúcar é prática cada vez mais difundida, por suas vantagens, tanto cultural como econômica (2, 5 e 7).

Já há algum tempo a Seção de Herbicidas vem se interessando no estudo do controle quimico das plantas daninhas infestantes dessa cultura (3). Diversos herbicidas residuais foram testados, destacando-se como promissores, atrazine e 2,4-D, entre outros.

Com o aparecimento de quatro outros herbicidas de ação residual, promissores para uso na cultura de canade-açúcar, foram conduzidos dois experimentos de campo em 1971/72, sendo os herbicidas testados em pré-emergência.

\section{MAT ERIAIS E MÉTODOS}

Em 1971 foram conduzidos dois ensaios de campo, idênticos, com apli- cação de herbicidas em pré-emergência em cana-de-açúcar, varie dade CB 41-76. Os ensaios foram instalados em Capivarí, SP e em Leme, SP, e os resultados das análises de amostras compostas de solos das áreas utilizadas, efetuadas pela Seção de Fertilidade de Solo, e de Pedologia, do Instituto Agronômico, encontram-se no Quadro 1.

O delineamento experimental escolhido foi o de blocos ao acaso, com 15 tratamentos repetidos quatro vezes. Parcelas com cinco linhas de cana-deaçúcar de 10,00 m de comprimento, espaçadas de $1,20 \mathrm{~m}$ em Capivari e $1,50 \mathrm{~m}$ em Leme. As áreas úteis de $36,00 \mathrm{~m}^{2} \mathrm{e}$ de $45,00 \mathrm{~m}^{2}$ respectivamente, eram formadas pelas três linhas centrais de cada parcela.

Os herbicidas seletivos testados encontravam-se em fase experimental por ocasião do desenvolvimento dos ensaios, sendo indicados pelos códigos RP 17.623 , R 7465, VCS 438 e A-3587.

A amostra experimental de RP $\left.17.623{ }^{1}{ }^{1}\right]$, fornecida pelo fabricante, é um concentrado emulsionável contendo $25 \%$ de oxadiazon, ou seja, de tertiobutil 2-(dicloro-2, 4 isopropiloxi-5-fenil)4-oxo5 oxadiasoline-1,3,4. Oxadiazon foi aplicado nas doses de $1,0 \mathrm{~kg}, 1,5 \mathrm{~kg}$ e $2,0 \mathrm{~kg} / \mathrm{ha}$.

R $7465\left[^{2}\right]$, fornecido pelo fabricante, é um pó molhável contendo $50 \%$ de

QUADRO 1 - Resultados das análises de solos realizados pela Seçâo de Fertiliaade de Solo e de Pedologia do Instituto Agronómico.

\begin{tabular}{lcr}
\hline & CAPIVARI & LEME \\
\hline pH & 6,10 & 6,30 \\
M.O. $(\%)$ & 1,54 & 3,65 \\
Argila $(\%)$ & 17,5 & 32,5 \\
Limo $(\%)$ & 8,7 & 7,5 \\
Areia fina $(\%)$ & 57,2 & 41,4 \\
Areia grossa $(\%)$ & 16,6 & 18,6 \\
Classe textural & fraco areno barrento & barrento \\
\hline
\end{tabular}

Produto comercial usado: ['] Ronstar

[ ${ }^{7}$ Devrinol 50 WP 
2-(a naftoxi)N,N-dietilpropionamida, cujo nome comum é napropamide. Nos ensaios foram usadas as doses de 2,0 $\mathrm{kg}, 2,5 \mathrm{~kg}$ e $3,0 \mathrm{~kg} / \mathrm{ha}$ de ingrediente ativo.

VCS 438 [3] é um pó molhável com $75 \%$ de methazole 2-(3,4-diclorofenil)-4metil-1,2,4-oxadiazolidine-3,5-dione. Foi usado a $3,0 \mathrm{~kg}, 3,5 \mathrm{~kg}$ e $4,0 \mathrm{~kg} / \mathrm{ha}$, de ingrediente ativo.

A-35-87[ $\left.{ }^{4}\right]$, é um pó molhável contendo $40 \%$ de 2 -cloro-4-etilamino-6-tertbutilamino-s-triazina e $40 \%$ de 2 -metoxi-4-etilamino-6-tert - butilamino-s-triazina. Foi usado a 2,0 kg, 3,0 kg e 4,0 $\mathrm{kg} / \mathrm{ha}$ de ingrediente ativo.

Como padrão foram empregados

2,4-D amina [5], e atrazine [e]. 2,4-D foi empregado na dose de $2,0 \mathrm{~kg} / \mathrm{ha}$ e a atrazine a $3,0 \mathrm{~kg} / \mathrm{ha}$.

Todos os herbicidas foram aplicados em pré-emergência, no dia seguinte ao plantio da cana-de-açúcar, em 30.09. 71 em Capivarí, e em 12.10.71 em Leme. As aplicações foram feitas com pulverizador es do tipo costal manual, com capacidade para 15 litros, munidos de bico de jato em leque, trabalhando a 35 $\mathrm{lb} /$ po $1^{2}$ de pressão, propiciando uma cobertura total da parcela, com um gasto de 5001 de calda/ha

Foi incluido um tratamento testemunha, capinado com enxada.

Para a avaliação da eficiência dos herbicidas experimentados foi considerada a porcentagem de controle das plantas infestantes, tomada em relação à testemunha. Para a obtenção da porcentagem de controle foi realizada contage $m$ das plantas daninhas aos 30 dias após a aplicação dos herbicidas, em quatro amostras por parcela, em área de $0,50 \mathrm{~m}^{2}(1,00 \times 0,50 \mathrm{~m})$ cada, representativa da infestação média. As principais plantas daninhas que formavam a infestação natural dos experimentos foram:
Monocotiledóneas:

Brachiaria plantaginea (Link) Hitch. capim-marmelada

Digitaria sanguinalis (L.) Scop. cam pim-de-colchão

Eleusine indica (L.) Gaertn. cam pim-pé-de-galinha

Dicotiledóneas:

Portulacca olerace a L.

beldroega

Sida spp

guanxumas

Foram feitas observações visuais sobre possiveis sintomas de fitotoxicidade causados pelos herbicidas à cultura, assim como, foi tomado o número de colmos por parcela aos 60 dias após a aplicação dos produtos. O corte da cana-de-açúcar foi feito somente no ensaio de Leme, em 18.08.72.

\section{RESULTADOS E DISCUSSÃO}

As porcentagens de controle das plantas daninhas obtidas encontram-se nos Quadros 2 e 3.

No Quadro 4 são apresentados os números de colmos e a produção de cana-de-açúcar, esta somente do ensaio de Leme, visto que em Capivari o experimento não teve continuidade por motivos alheios à vontade dos autores.

A análise estatística das médias dos dados de controle geral das plantas daninhas, depois de transformadas para arcsen raíz quadrada de porcentagem, no ensaio de Capivarí, mostraram que oxadiazon, nas três doses testadas, methaloze a 3,5 e $4,0 \mathrm{~kg} / \mathrm{ha}$ e A-3587 a 3,0 e $4,0 \mathrm{~kg} / \mathrm{ha}$ apresentaram os melhores resultados, com indices de controle geral superiores a $80 \%$. Esses herbicidas ofereceram um controle acima de $90 \%$ para cada uma das principais plantas daninhas que apareceram

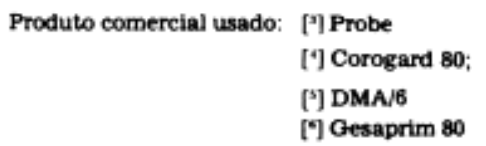




\begin{tabular}{|c|c|c|c|c|c|c|c|}
\hline \multirow[b]{2}{*}{ Herbicidas } & \multirow{2}{*}{$\begin{array}{l}\text { Ingrediente } \\
\text { ativo } \\
(\mathbf{k g} / \mathbf{h a})\end{array}$} & \multicolumn{5}{|c|}{ Porcentagem de controle } & \multirow{2}{*}{$\begin{array}{l}\text { Média de } \\
\text { controle geral } \\
\text { (transf. arc sen } \sqrt{\%} \text { ) }\end{array}$} \\
\hline & & $\begin{array}{l}\text { Brachiaria } \\
\text { plantaginea }\end{array}$ & $\begin{array}{l}\text { Digitaria } \\
\text { sanguinalis }\end{array}$ & $\begin{array}{l}\text { Eleusine } \\
\text { indica }\end{array}$ & $\begin{array}{l}\text { Portulacca } \\
\text { oleraced }\end{array}$ & Geral & \\
\hline Oxadiazon & $\begin{array}{l}1,0 \\
1,5 \\
2,0\end{array}$ & $\begin{array}{l}97,4 \\
98,1 \\
98,9\end{array}$ & $\begin{array}{l}95,0 \\
97,7 \\
96,5\end{array}$ & $\begin{array}{l}98,3 \\
99,1 \\
99,1\end{array}$ & $\begin{array}{l}100,0 \\
100,0 \\
100,0\end{array}$ & $\begin{array}{l}97,0 \\
98,2 \\
98,5\end{array}$ & $\begin{array}{ll}81,5 & \text { cd } \\
83,1 & \text { d } \\
84,5 & \text { d }\end{array}$ \\
\hline Napropamide & $\begin{array}{l}2,0 \\
2,5 \\
3,0\end{array}$ & $\begin{array}{l}84,9 \\
85,5 \\
89,3\end{array}$ & $\begin{array}{l}87,9 \\
98,0 \\
98,0\end{array}$ & $\begin{array}{l}96,6 \\
97,5 \\
97,5\end{array}$ & $\begin{array}{l}60,0 \\
60,0 \\
84,4\end{array}$ & $\begin{array}{l}85,1 \\
87,7 \\
91,5\end{array}$ & $\begin{array}{l}67,5 \text { b } \\
69,7 \text { bc } \\
73,4 \text { bod }\end{array}$ \\
\hline Methazole & $\begin{array}{l}3,0 \\
3,5 \\
4,0\end{array}$ & $\begin{array}{l}98,1 \\
98,7 \\
99,3\end{array}$ & $\begin{array}{l}92,2 \\
92,9 \\
97,2\end{array}$ & $\begin{array}{l}68,9 \\
93,3 \\
93,3\end{array}$ & $\begin{array}{l}98,9 \\
98,9 \\
98,9\end{array}$ & $\begin{array}{l}94,9 \\
97,1 \\
98,5\end{array}$ & $\begin{array}{rr}77,6 & \text { bod } \\
81,7 & \text { cd } \\
83,9 & \text { d }\end{array}$ \\
\hline$A-3587$ & $\begin{array}{l}2,0 \\
3,0 \\
4,0\end{array}$ & $\begin{array}{l}98,1 \\
98,0 \\
98,4\end{array}$ & $\begin{array}{l}92,9 \\
92,9 \\
95,5\end{array}$ & $\begin{array}{r}79,8 \\
99,1 \\
100,0\end{array}$ & $\begin{array}{r}100,0 \\
98,9 \\
100,0\end{array}$ & $\begin{array}{l}95,9 \\
97,0 \\
98,0\end{array}$ & $\begin{array}{r}79,3 \text { bcd } \\
80,7 \text { cd } \\
82,5 \text { d }\end{array}$ \\
\hline $2,4-\mathrm{D}$ & 2,0 & 54,8 & 75,3 & 68,1 & 86,7 & 61,2 & 51,5 a \\
\hline Atrazine & 3,0 & 95,0 & 86,1 & 100,0 & 98,9 & 93,6 & 75,8 bod \\
\hline \multicolumn{2}{|c|}{$\begin{array}{l}\text { Testemunha (n.o de } \\
\text { plantas daninhas } \mathrm{p} / \mathrm{m}^{2} \text { ) }\end{array}$} & 1213 & 397 & 119 & 90 & 1891 & - \\
\hline $\begin{array}{l}\text { lias assinalada } \\
\text { nificativas ao }\end{array}$ & $\begin{array}{l}\text { as pela n } \\
\text { nivel do }\end{array}$ & & & & & $\begin{array}{l}\text { C.V. } \quad(\%) \\
\text { F. } \\
\text { D.M.S. }\end{array}$ & $\begin{array}{l}5,6 \\
14,39^{*} \\
12,2\end{array}$ \\
\hline
\end{tabular}

no experimento. Com controle geral um pouco inferior ao daqueles herbicidas anteriormente citados, aparecem: napropamide a $3,0 \mathrm{~kg} / \mathrm{ha}$, methazole quando empregado a $3,0 \mathrm{~kg} / \mathrm{ha}$ e A3587 a $2,0 \mathrm{~kg} / \mathrm{ha}$, além de atrazine. Napropamide a $3,0 \mathrm{~kg} /$ ha não conseguiu indices superiores a $90,0 \%$ para $B$. plantaginea e $P$. oleracea; methazole a $3,0 \mathrm{~kg} / \mathrm{ha}$ controlou $E$. indica em apenas $68,9 \%$; e, A-3587 a $2,0 \mathrm{~kg} / \mathrm{ha}$ também não foi eficiente no controle de $E$. indica, com Índice de apenas 79,8\%.

Os resultados oferecidos pelos herbicidas testados, no ensaio de Leme, foram inferiores aos de Capivari. Atribui-se essa menor porcentagem de controle aos teores de argila e de matéria orgânica, mais elevados no solo de Leme, e também, ao aparecimento de Si-da spp, plantas daninhas mais dificeis de serem controladas pelos herbicidas usuais. Neste município, oxadiazon, apenas na dose mais elevada, de $2,0 \mathrm{~kg} /$ ha, conseguiu bons resultados de controle. A-3587, a $3,0 \mathrm{~kg}$ e $4,0 \mathrm{~kg} / \mathrm{ha}$, mesmo nestas condições também ofereceu bons resultados de controle de $D$. sanguinalis e Sida spp, que foram as principais plantas daninhas incidentes. Estão em categorias imediatamente infe rior: atrazine a $3,0 \mathrm{~kg} / \mathrm{ha}$, oxadiazon a $1,5 \mathrm{~kg} / \mathrm{h}$ a e $2,4-\mathrm{D}$ a $2,0 \mathrm{~kg} / \mathrm{ha}$.

Atrazine, usado como padrão, apresentou os resultados esperados. Whitehead (8), em experimento conduzido na Africa do Sul, também encontrou bons resultados de controle de plantas daninhas até a cana-de-açúcar cobrir todo o solo, com atrazine, o qual superou 2,4-D. Os resultados com 2,4$\mathrm{D}$, também usado como padrão, foram inferiores aos esperados, principalmente no experimento de Capivari. Porém, na época da aplicação dos herbicidas o solo encontrava-se bastante úmido, continuando nesse estado por mais do que sete dias, devido a elevada queda plu- 
QUADRO 3 - Porcentagem de controle de plantas daninhas, 30 dias após a aplicaçáo de herbicidas em pré-emergência e médias de controle geral com dados transformados para are sen $\sqrt{ } \%$, em cana-de-açúcar, em Leme, SP, em 1971/72. Os dados sảo médias de quatro repetiçōes.

\begin{tabular}{|c|c|c|c|c|c|}
\hline \multirow[b]{2}{*}{ Herbicidas } & \multirow{2}{*}{$\begin{array}{l}\text { Ingrediente } \\
\text { ativo } \\
\text { (kg/ha) }\end{array}$} & \multicolumn{2}{|c|}{ Porcentagem de controle } & \multirow[b]{2}{*}{ Geral } & \multirow{2}{*}{$\begin{array}{c}\text { Média de } \\
\text { controle geral } \\
\text { (transf. arc. sen } \sqrt{\%})\end{array}$} \\
\hline & & $\begin{array}{l}\text { Digitaria } \\
\text { sanguinalis }\end{array}$ & Sida spp & & \\
\hline Oxadiazon & $\begin{array}{l}1,0 \\
1,5 \\
2,0\end{array}$ & $\begin{array}{l}95,1 \\
98,8 \\
98,8\end{array}$ & $\begin{array}{l}50,8 \\
62,7 \\
88,9\end{array}$ & $\begin{array}{l}68,3 \\
76,9 \\
92,6\end{array}$ & $\begin{array}{cc}55,8 & \text { bcde } \\
61.4 & \text { ef } \\
75,2 & \text { gh }\end{array}$ \\
\hline Napropamide & $\begin{array}{l}2,0 \\
2,5 \\
3,0\end{array}$ & $\begin{array}{l}87,8 \\
93,9 \\
95,1\end{array}$ & $\begin{array}{r}0,0 \\
27,0 \\
28,6\end{array}$ & $\begin{array}{l}32,8 \\
53,4 \\
54,8\end{array}$ & $\begin{array}{l}34,8 \text { a } \\
47,0 \text { b } \\
47,8 \text { bc }\end{array}$ \\
\hline Methazole & $\begin{array}{l}3,0 \\
3,5 \\
4,0\end{array}$ & $\begin{array}{l}64,6 \\
78,0 \\
87,8\end{array}$ & $\begin{array}{l}55,5 \\
62,7 \\
61,1\end{array}$ & $\begin{array}{l}59,1 \\
68,7 \\
71,6\end{array}$ & $\begin{array}{l}50,3 \text { bod } \\
56,0 \text { bode } \\
57,8 \text { cdef }\end{array}$ \\
\hline A-3587 & $\begin{array}{l}2,0 \\
3,0 \\
4,0\end{array}$ & $\begin{array}{l}84,1 \\
92,7 \\
97,6\end{array}$ & $\begin{array}{l}61,1 \\
88,1 \\
96,8\end{array}$ & $\begin{array}{l}70,2 \\
89,9 \\
97,1\end{array}$ & $\begin{array}{rr}57,0 & \text { bcde } \\
71,8 & \text { gh } \\
81,7 & \text { h }\end{array}$ \\
\hline $2,4-\mathrm{D}$ & 2,0 & 78,0 & 68,2 & 74,0 & 59,4 \\
\hline Atrazine & 3,0 & 86,6 & 84,1 & 85,1 & 67,4 \\
\hline \multicolumn{2}{|c|}{$\begin{array}{r}\text { Testemunha (n.o de plantas } \\
\text { daninhas } \mathrm{p} / \mathrm{m}^{2} \text { ) } \\
\end{array}$} & 82 & 126 & 208 & - \\
\hline & & & & $\begin{array}{l}\text { C.V. } \quad(\%) \\
\text { F. } \\
\text { D.M.S. }\end{array}$ & $\begin{array}{l}6,7 \\
38,65^{*} \\
10,2 \\
\end{array}$ \\
\hline
\end{tabular}

Médias assinaladas pela mesma letra não apresentam diferenças significativas ao nível de $5 \%\left({ }^{*}\right)$, pelo teste de Tukey.

QUADRO 4 - Médias do número de colmos e de produçáo (kg/tratamento) de cana-de-açúcar dos ensaios em pré-emergência, em Capivari, SP e Leme, SP, em 1971/72. Os dados sáo médias de quatro repetiçóes.

\begin{tabular}{|c|c|c|c|c|c|}
\hline \multirow[b]{2}{*}{ Herbicidas } & \multirow{2}{*}{$\begin{array}{l}\text { Ingrediente } \\
\text { ativo } \\
\text { (kg/ha) }\end{array}$} & \multicolumn{2}{|c|}{ Capivari } & \multicolumn{2}{|c|}{ Leme } \\
\hline & & $\begin{array}{l}\text { Médias de n.o } \\
\text { de colmos }\end{array}$ & $\begin{array}{l}\text { Médias de pro- } \\
\text { dução }\end{array}$ & $\begin{array}{l}\text { Médias de n.o } \\
\text { de colmos }\end{array}$ & $\begin{array}{l}\text { Médias de pro- } \\
\text { dução (x 1000) }\end{array}$ \\
\hline Oxadiazon & $\begin{array}{l}1,0 \\
1,5 \\
2,0\end{array}$ & $\begin{array}{l}752,0 \mathrm{a} \\
727,0 \mathrm{a} \\
761,0 \mathrm{a}\end{array}$ & $\begin{array}{l}- \\
-\end{array}$ & $\begin{array}{l}749,0 \\
798,0 \\
721,0\end{array}$ & $\begin{array}{l}2,0 \mathrm{ab} \\
2,1 \mathrm{bc} \\
2,1 \mathrm{bc}\end{array}$ \\
\hline Napropamide & $\begin{array}{l}2,0 \\
2,5 \\
3,0\end{array}$ & $\begin{array}{l}631,0 \mathrm{a} \\
705,0 \mathrm{a} \\
768,0 \mathrm{a}\end{array}$ & $\begin{array}{l}- \\
-\end{array}$ & $\begin{array}{l}742,2 \\
703,0 \\
804,0\end{array}$ & $\begin{array}{l}2,1 \mathrm{bc} \\
1,8 \mathrm{a} \\
2,0 \mathrm{ab}\end{array}$ \\
\hline Methazole & $\begin{array}{l}3,0 \\
3,5 \\
4,0\end{array}$ & $\begin{array}{l}800,0 \mathrm{~b} \\
802,0 \mathrm{~b} \\
735,0 \mathrm{a}\end{array}$ & $\begin{array}{l}- \\
- \\
-\end{array}$ & $\begin{array}{l}789,0 \\
818,0 \\
817,0\end{array}$ & $\begin{array}{l}1,9 \mathrm{ab} \\
2,1 \mathrm{ab} \\
2,2 \mathrm{c}\end{array}$ \\
\hline A-3587 & $\begin{array}{l}2,0 \\
3,0 \\
4,0\end{array}$ & $\begin{array}{l}729,0 \text { a } \\
719,0 \text { a } \\
837,0 \text { b }\end{array}$ & $\begin{array}{l}- \\
-\end{array}$ & $\begin{array}{l}699,0 \\
723,0 \\
744,0\end{array}$ & $\begin{array}{l}2,1 \mathrm{bc} \\
1,9 \mathrm{ab} \\
1,9 \mathrm{ab}\end{array}$ \\
\hline 2,4-D & 2,0 & 677,0 a & - & 771,0 & $2,0 \mathrm{ab}$ \\
\hline Atrazine & 3,0 & 681,0 a & - & 796,0 & 2,1 bc \\
\hline Testemunha & - & 667,0 a & - & 791,0 & 1,8 a \\
\hline $\begin{array}{l}\text { C.V. } \quad(\%) \\
\text { F. } \\
\text { D.M.S. }\end{array}$ & & $\begin{array}{c}15,6 \\
7,55^{*} \\
104,3 \\
\end{array}$ & & $\begin{array}{l}6,1 \\
3,08 \text { n.s. }\end{array}$ & $\begin{array}{l}4,2 \\
6,97^{*} \\
0,2 \\
\end{array}$ \\
\hline
\end{tabular}

Médias assinaladas pela mesma letra não apresentam diferenças significativas ao nível de $5 \%\left({ }^{*}\right)$, pelo teste de Tukey. 
viométrica no período. Essa condição pode ter prejudicado a ação de 2,4-D, considerando que esse herbicida é de fácil percolação. Alguns autores têm encontrado resultados diferentes de controle de plantas daninhas com 2,4-D. Assim é que Hughan \& Booth (4), em Zâmbia, em ensaios com aplicações de herbicidas em pré e pós-emergência, encontraram bons resultados de controle de gramineas, exceto para $E$. indica, e de folhas largas por 2,4-D em Nakambala, enquanto que em Kafué os resultados foram inferiores. Oliveira et al. (5) compararam a ação de diuron, terbacil, atrazine, 2,4-D, fluometuron e as misturas de diuron com terbacil e com 2,4-D e de terbacil com atrazine, encontrando os melhores resultados de controle geral, entre 85 e 90\%, para as misturas, seguidas, com controle de 80 a $85 \%$, de diuron. Atrazine e fluometuron conseguiram um controle geral de 75 a $80 \%$, e 2,4-D amina apresentou também os piores resultados, com indices variando de 70 a $75 \%$.

Nenhum dos produtos testados foi prejudicial ao perfilhamento e à produção de cana-de-açúcar. As produções obtidas, quando analisadas estatísticamente, mostraram diferenças significativas ao nivel de $5 \%$. Todos os tratamentos com herbicidas mostraram-se superiores à testemunha. Azzi \& Fernandes (1), estudando a possibilidade de influência dos herbicidas ametrine, atrazine, diuron e 2,4-D na produção de cana-de-açúcar, também concluiram pela influência no seu aumento, tanto nas aplicações em pré como em pósemergência. Os maiores estímulos foram encontrados por atrazine a $4,0 \mathrm{~kg} / \mathrm{ha}$ em pré-emergência, e a $2,0 \mathrm{~kg} / \mathrm{ha} \mathrm{em}$ pós-emergência.

Dos resultados obtidos nos experimentos relatados pode-se tirar as seguintes conclusões:

1 - Em solo franco areno barrento, oxadiazon a $1,0 \mathrm{~kg}, 1,5 \mathrm{~kg}$ e $2,0 \mathrm{~kg} / \mathrm{ha}$, methazole a $3,5 \mathrm{~kg}$ e $4,0 \mathrm{~kg} / \mathrm{ha}$ e A-3587 a $3,0 \mathrm{~kg}$ e $4,0 \mathrm{~kg} / \mathrm{ha}$ e, em solo barrento,
A-3587 a 3,0 kg e 4,0 kg/ ha e oxadiazon a $2,0 \mathrm{~kg} / \mathrm{ha}$ apresentaram os melhores resultados de controle das plantas dani nhas incidentes nos ensaios.

2 - Atrazine usado como padrão, apresentou um controle acima de $80 \%$ para todas as plantas daninhas dos dois experimentos. 2,4-D, também usado como padrão, controlou apenas $P$. oleracea, e ofereceu controle geral entre 50 e $60 \%$.

3 - Nenhum dos herbicidas estudados, nas doses em que foram testados, mostrou-se prejudicial ao perfilhamento, ao desenvolvimento normal e à produção da cultura.

\section{LITERA TURA CITADA}

1. Azzi, G.M. \& Fernandes, J. Estímulo em canade-açúcar tratada com ametrin, atrazin, diuron e 2,4-D. In: Sem. Bras. Herb. Ervas Dan., 8.', Botucatu, 1970. Resumos, p.V-07.

2. Baroni, O. Cultivo químico e cultivo mecânico em cultura de cana-de-açúcar - comparaçáo de custos. In: Sem. Bras. Herb. Ervas Dan., 2., Belo Horizonte, 1958. Anais, p.229-35.

3. Cruz, L.S.P. \& Leiderman, L. Nova triazina para cana-de-açúcar (Saccharum hibridus). In: Sem. Bras. Herb. Ervas Dan., 9.', Campinas, 1972. Resumos, p. 28.

4. Hughan, D.S. \& Booth, D.R.C. Weed control on a newly developing estate at Mazabuka, Zambia. In: S. Afri. Sug. Techmol. Ass., Proc. 312-14 e 16, 1966.

5. Oliveira, O.; Gregori, R. \& Paranhos, S.B. Experiência com herbicidas empregados a sós e combinados, em aplicaçóes de pré e pósemergencia em cana-de-açúcar. In: Sem. Bras. Herb. Ervas Dan., 6., Sete Lagoas, 1966. Anais. p. 211-18.

6. Oliveira, O.; Fernandes, R.S.; Gregori, R.; Paranhos, S.B. \& Espironelo, A. Emprego de herbicidas na cana-de-açúcar com a finalidade de controlar as ervas daninhas e verificar a sensibilidade de variedades. In: Sem. Bras. Herb. Ervas Dan, 8. , Botucatu, 1970. Resumos, p. V-03.

7. Souza, H.D. Aspectos económicos da aplicaçáo de Herbicidas em cana-de-açúcar. In: Sem. Bras. Herb. Ervas Dan., 2.*, Belo Horizonte, 1958. Anais, p. 237-42.

8. Whitehead, C. Sugarcane in South Africa - its production and management. Bull. $\mathbf{S}$. Afr. Sug. Ass. Exp. Stn., 1, 1965. 24p. 